\title{
Bio-orthogonal Red and Far-Red Fluorogenic Probes for Wash-Free Live-Cell and Super-resolution Microscopy
}

Philipp Werther, ${ }^{\# \#}$ Klaus Yserentant, ${ }^{\# \#}$ Felix Braun, Kristin Grußmayer, Vytautas Navikas, Miao Yu, Zhibin Zhang, Michael J. Ziegler, Christoph Mayer, Antoni J. Gralak, Marvin Busch, Weijie Chi, Frank Rominger, Aleksandra Radenovic, Xiaogang Liu, Edward A. Lemke, Tiago Buckup, Dirk-Peter Herten, * and Richard Wombacher*

Cite This: ACS Cent. Sci. 2021, 7, 1561-1571

Read Online

ACCESS | Lill Metrics \& More | 期 Article Recommendations

Supporting Information

ABSTRACT: Small-molecule fluorophores enable the observation of biomolecules in their native context with fluorescence microscopy. Specific labeling via bio-orthogonal tetrazine chemistry combines minimal label size with rapid labeling kinetics. At the same time, fluorogenic tetrazine-dye conjugates exhibit efficient quenching of dyes prior to target binding. However, live-cell compatible long-wavelength fluorophores with strong fluorogenicity have been difficult to realize. Here, we report close proximity tetrazine-dye conjugates with minimal distance between tetrazine and the fluorophore. Two synthetic routes give access to a series of cell-permeable and -impermeable dyes including highly

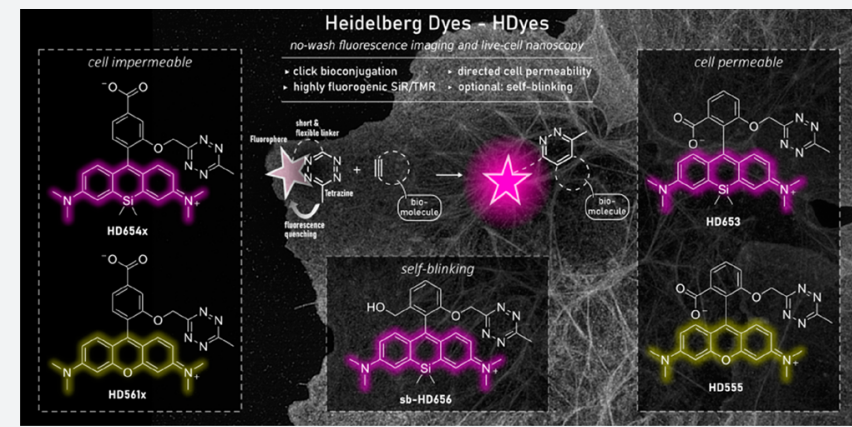
fluorogenic far-red emitting derivatives with electron exchange as the dominant excited-state quenching mechanism. We demonstrate their potential for live-cell imaging in combination with unnatural amino acids, wash-free multicolor and super-resolution STED, and SOFI imaging. These dyes pave the way for advanced fluorescence imaging of biomolecules with minimal label size.

\section{INTRODUCTION}

Over the last decades, fluorescence microscopy has become an indispensable tool to study biomolecules in their native cellular environment. A fundamental challenge is to achieve specific fluorescent labeling of biomolecules while minimizing the size of the label. This is crucial to reduce the perturbation of the biomolecule's behavior as well as to avoid distortions by linkage errors in super-resolution microscopy. ${ }^{1}$ In recent years, bio-orthogonal chemistry has become an important approach to selectively introduce small organic fluorophores. Various strategies have been developed to label proteins, ${ }^{2,3}$ nucleic acids, ${ }^{4,5}$ sugars, ${ }^{6,7}$ or lipids. ${ }^{8,9}$ With the advancement of bioorthogonal chemistries, probe designs have been reported where bond formation is accompanied by fluorescence enhancement. ${ }^{10,11}$ This so-called fluorogenic effect can substantially improve the signal-to-background ratio in fluorescence microscopy and can allow no-wash experiments. ${ }^{12-15}$ In particular, quenched tetrazine dyes that react in inverse electron demand Diels-Alder $\left(\mathrm{DA}_{\text {inv }}\right)$ reactions bear great potential as fluorogenic bio-orthogonal labels due to fast kinetics and high biocompatibility. ${ }^{16}$ Previous work has shown that the quenching efficiency in tetrazine dyes determines the fluorescence turn-on of the probe upon reaction and is strongly dependent on the interchromophore distance between tetrazine and fluorophore. ${ }^{17-19}$ Tetrazine-dye designs with short interchromophore distances, however, show only very low quenching efficiencies for far-red-shifted fluorophores compared to their blue-shifted analogues. ${ }^{14,20,21}$

Beyond fluorogenicity and bioconjugation functionality, fluorescent labels need to exhibit high water solubility and should not aggregate or bind unspecifically to cellular structures. Moreover, cell permeability, high fluorescence brightness, and high photostability are crucial for long-term observation of intracellular targets. Rhodamines and silicon rhodamines ( $\mathrm{SiRs}$ ), both xanthene-type dyes, meet these demands and are widely used in super-resolution fluorescence microscopy techniques like single-molecule localization microscopy (SMLM) $)^{22,23}$ or stimulated emission depletion microscopy (STED). ${ }^{24,25}$ Different structural designs for fluorogenic tetrazine conjugates have been developed for both dye classes. ${ }^{14,26,27}$ For rhodamines, a fluorescence enhancement up to 76 -fold has been reported. ${ }^{26}$ However,

Received: June 9, 2021

Published: August 20, 2021 
a
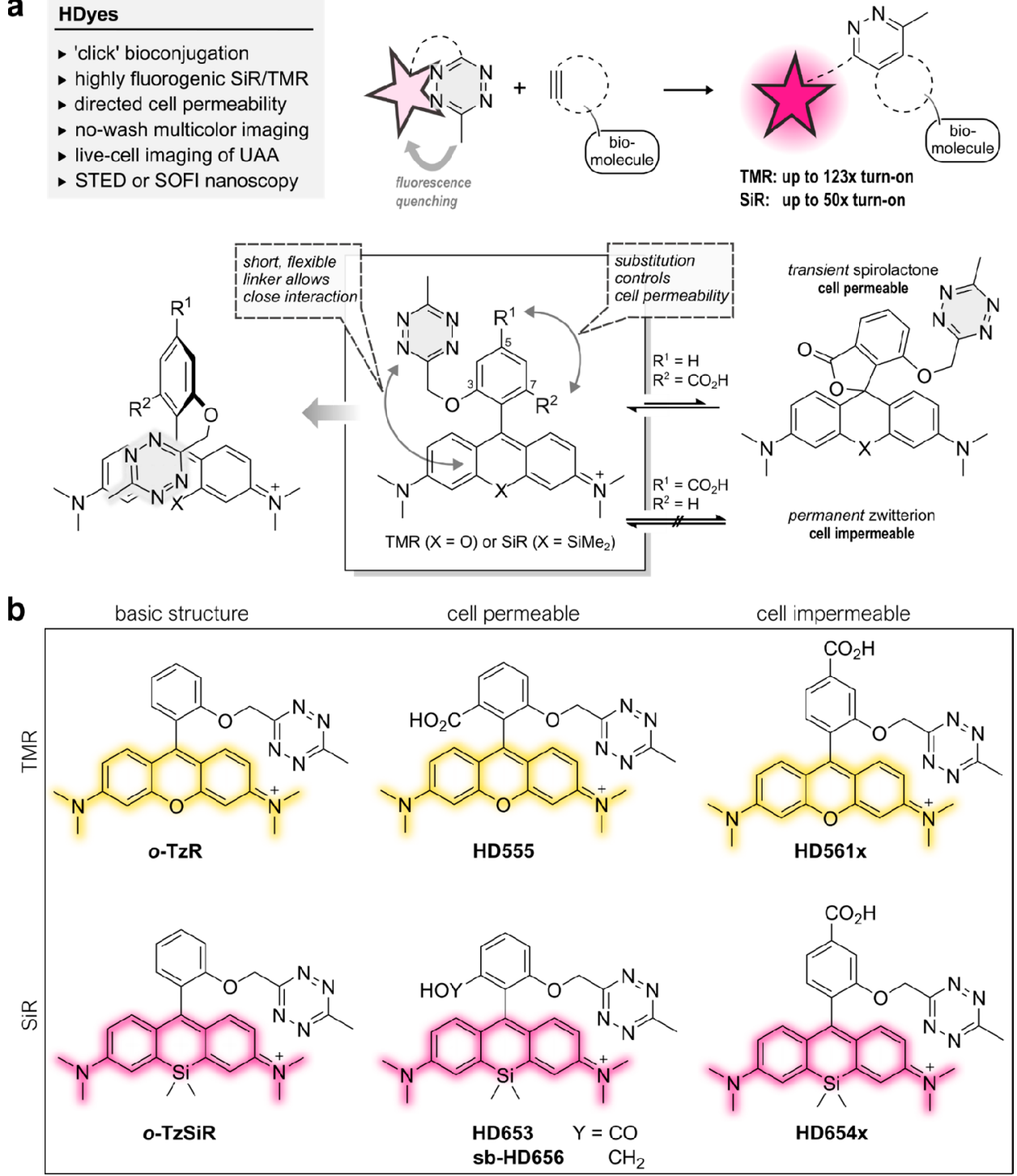

Figure 1. Design rationale for close proximity quenched fluorogenic probes. (a) Tetrazine dyes can be conjugated to dienophile-modified biomolecules accompanied by an increase in fluorescence intensity. The new design allows a close fluorophore-tetrazine interaction, resulting in efficient quenching and high fluorescence turn-on. Cell permeability is controlled by varying the position of a $\mathrm{CO}_{2} \mathrm{H}$ substituent. (b) Overview of fluorogenic TMR and SiR tetrazine probes (HDyes) with distinct cell permeability.

so far, quenched $\mathrm{SiR}$-tetrazine conjugates suffer from moderate fluorescence enhancements ${ }^{14}$ or do not allow livecell imaging. ${ }^{27}$

Here, we report a novel design concept for tetrazine-dye conjugates that enables highly efficient quenching for rhodamines and SiRs resulting in high fluorescence enhancement upon $\mathrm{DA}_{\mathrm{inv}}$. We have thoroughly characterized the spectroscopic properties of the tetrazine-dyes and found evidence of Dexter exchange as the underlying fluorescence quenching mechanism. The tetrazine-dye conjugates that we name HDyes ("Heidelberg Dyes") exhibit excellent properties for a number of live-cell applications including the targeting of unnatural amino acids (UAAs) and allow extra- and intracellular multicolor wash-free labeling. Finally, we demonstrate that bio-orthogonal labeling with HDyes enables live-cell STED as well as super-resolution optical fluctuation imaging (SOFI).

\section{RESULTS AND DISCUSSION}

Design and Synthesis of Close Proximity Quenched Fluorogenic HDyes. The main challenge in the development of fluorogenic probes for bio-orthogonal chemistry is to accommodate both high fluorogenicity upon target binding and favorable properties for live-cell imaging in one molecular structure. Generally, an ideal concept enables further structural modification of the fluorophore to provide distinct molecular functionalities for specialized applications. In the case of fluorogenic tetrazine dyes, efficient fluorescence quenching is particularly difficult to achieve with red-shifted fluorophores. ${ }^{20}$ To address this problem, we designed tetramethylrhodamine (TMR) - and SiR-tetrazine conjugates with minimized interchromophore distances. In this design, the tetrazine is placed in close proximity to the fluorophore by an unconjugated chemical linkage in the ortho-position of the phenyl ring pendant to the xanthene core (Figure 1a). We reasoned that the short and flexible oxymethyl linker would lead to a stacked conformation of the fluorophore and tetrazine 
Scheme 1. Synthesis of HDyes ${ }^{a}$

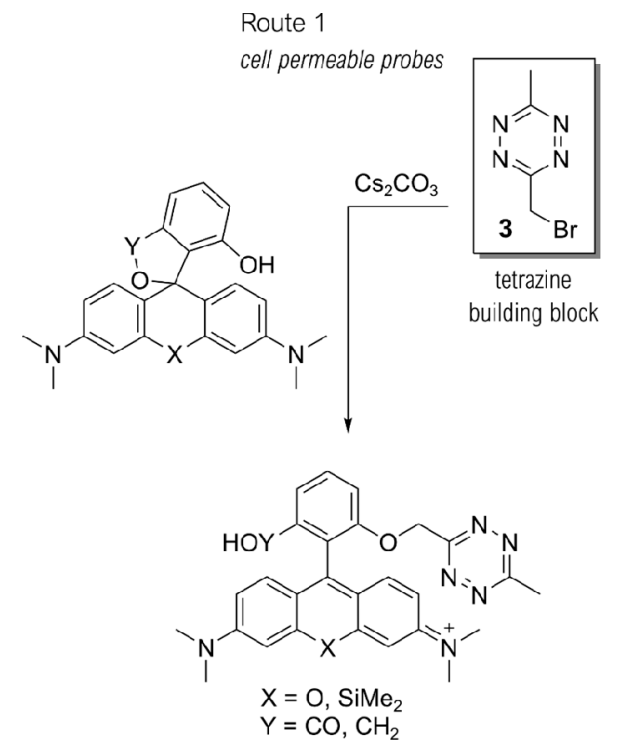

Route 2
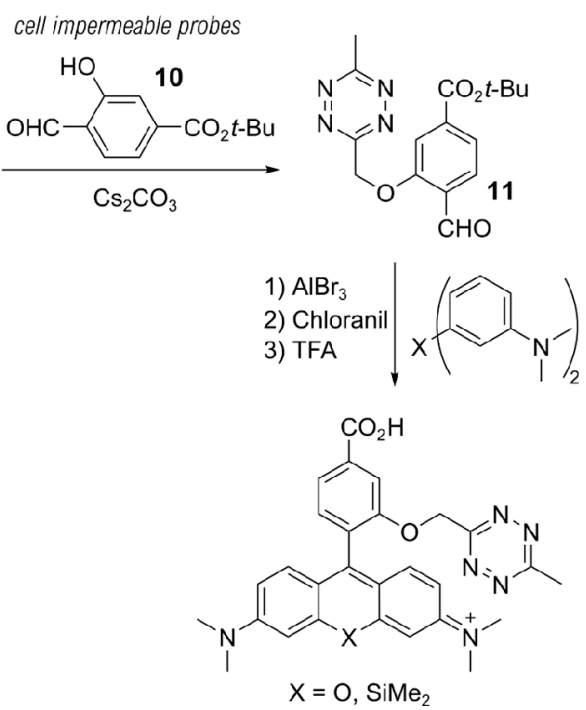

${ }^{a_{T}}$ Two complementary synthetic routes provide access to ortho-oxymethyl-linked SiR and TMR tetrazine probes with distinct cell permeability. The key compound for tetrazine derivatization in both routes is the bromomethyl tetrazine building block 3 .

as was suggested in previous work from Beliu et al. on oxazine and $\mathrm{SiR}$ dyes. ${ }^{28}$ As a consequence of this shortened bichromophoric linkage, the stacked conformation could become more likely, which in turn would improve fluorescence quenching. Furthermore, building on literature reports, we aimed to control cell permeability by variation of additional phenyl ring substitutions. It has been shown that a carboxylic acid substituent in the ortho-position results in high cell permeability owing to the formation of a transient uncharged spirolactone. ${ }^{12,13}$ We anticipated that a carboxylic acid substituent in the para-position lacking the possibility of spirolactonization yields a permanent zwitterionic, thus cellimpermeable dye.

To realize such cell-permeable and -impermeable $(\mathrm{Si})-$ rhodamine-tetrazines, we developed synthetic routes using bromomethyl tetrazine building block 3 (Scheme 1, see Schemes S1-4 and Table S1 for detailed description). We synthesized tetrazine benzaldehyde $\mathbf{1 1}$ from 3 via Williamson ether synthesis and used them in Friedel-Crafts-reactions ${ }^{14}$ to create 3,5-substituted $(\mathrm{Si})$-rhodamines (Route 2). Subsequent tert-butyl ester cleavage afforded cell-impermeable HDyes, HD561x and HD654x (Figure 1b). However, corresponding cell-permeable 3,7-substituted derivatives were not accessible through this route presumably due to inactivation of the benzaldehyde intermediate by hemiacetal formation and problematic ester cleavages. Yet, we were able to obtain phenolic $(\mathrm{Si})$-rhodamines by addition of bisaryllithium intermediates to phthalic anhydrides ${ }^{29}$ or phthalides and subsequent MOM deprotection (Route 1, Schemes 1 and S4). These were reacted with 3 in Williamson ether syntheses to furnish the respective $7-\mathrm{CO}_{2} \mathrm{H}$ cell-permeable HDyes HD555, HD653, and sb-HD656 (Figure 1b).

Photophysical Characterization and Investigation of the Quenching Mechanism. Understanding the quenching mechanism of fluorogenic probes is of particular importance for the experimental application and future developments in the field. Therefore, we studied the photophysical properties and the quenching mechanism of ortho-oxymethyl-linked tetrazine rhodamines using time-resolved spectroscopy. We synthesized the basic structure $\boldsymbol{o}$-TzR and found that it is highly quenched, with a fluorescence quantum yield $\left(\Phi_{\mathrm{F}}\right)$ of $0.3 \%$. In addition to $\boldsymbol{o}$-TzR, we synthesized regioisomers $\boldsymbol{m}$ and $p$-TzR where the oxymethyl-linked tetrazine is in the metaand para-positions, respectively (Figure 2a, Scheme S3, Table S2). Further, we reacted $\boldsymbol{o}$-TzR with (bicyclo[6.1.0]non-4-yn9-yl)methanol (BCN) to isolate the cycloadduct 26 and synthesized the structurally related methoxy-substituted $\mathbf{2 5}$, both serving as unquenched reference dyes (Figure 2a). We obtained the intramolecular tetrazine-xanthene distances $(R)$ for $o / m / p$-TzR from geometry-optimized structures using DFT calculations (Figure 2b). These computational results confirmed our hypothesis that o-TzR affords a stacked tetrazine-fluorophore conformation with a smaller intramolecular distance between tetrazine and the fluorophore than those of $m$-TzR and $p$-TzR.

We then studied the distance-dependence of the quenching by recording femtosecond transient absorption (TA) spectra with a variable probe delay after a $20 \mathrm{fs}$ excitation pulse at 575 $\mathrm{nm}$. We found that all compounds show ground-state bleach (GSB) and stimulated emission (SE) bands with a maximum at $560 \mathrm{~nm}$ (Figures S1, S2 and Note S1). The absorbance at $560 \mathrm{~nm}$ in dependence of the probe delay shows the different decays of all five compounds (Figure 2c). Reference compounds 25 and 26 lacking a quenching group showed a minor signal recovery within 1.3 ps followed by a very slow decay compatible with their emission characteristics. In contrast, the TA signal recovery was strongly accelerated for tetrazine regioisomers $o / m / p$-TzR: While in the case of $m / p$ TzR, the TA signal decayed almost to half of its initial amplitude after 16 ps, it was quenched to below $20 \%$ of the initial amplitude for $\boldsymbol{o}$-TzR (Figures S1, S2). Accordingly, $\boldsymbol{m}$ and $\boldsymbol{p}$-TzR exhibited substantially lower turn-on in $\mathrm{DA}_{\mathrm{inv}}$ of 6.9- and 8.5-fold, respectively (Figure S4, Table S1). For a quantitative assessment of the TA data, we performed global target analysis with a sequential model to the full data set (Figure S3 and Note S2). The fast recovery with a time constant $\tau_{2}$ of $1.56( \pm 0.06)$ ps for $\boldsymbol{o}$-TzR confirmed an efficient quenching compared to $m$-TzR and $p$-TzR with $\tau_{2}$ of 

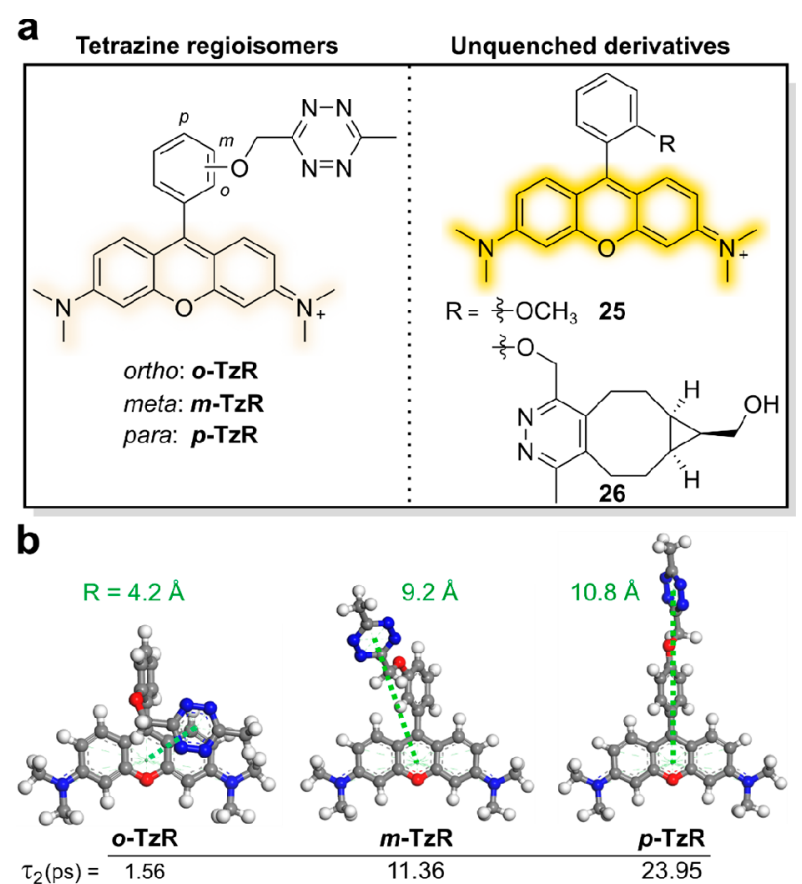

c
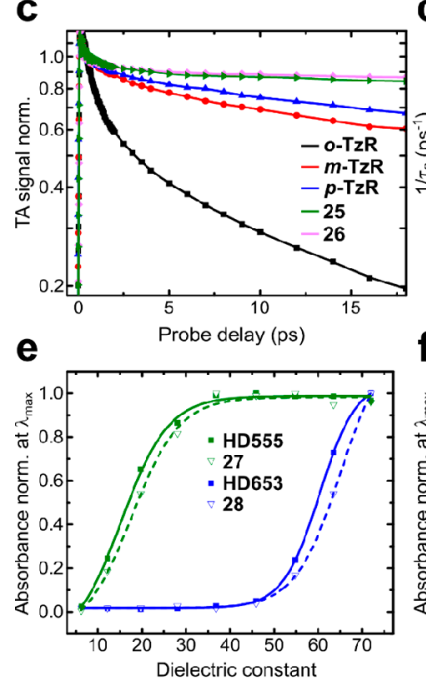

d

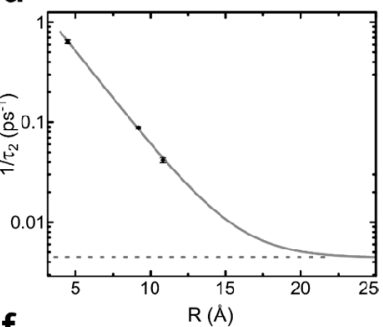

$f$

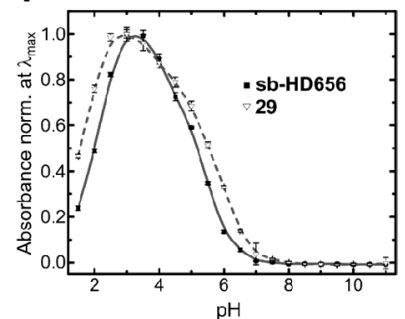

Figure 2. Fluorescence quenching and physicochemical properties of HDyes. (a-d) Time-resolved spectroscopy to study fluorescence quenching: (a) Structures of tetrazine rhodamine regioisomers and unquenched reference dyes. (b) DFT-optimized structures of $\boldsymbol{o} / \mathbf{m} / \mathbf{p}$ TzR, respective tetrazine-xanthene intramolecular distances $(R)$, and transient absorption decay times $\tau_{2}$. (c) Selected transient absorption traces at $560 \mathrm{~nm}$ (max. of GSB/SE). Data was normalized at a probe delay of 0.5 ps to avoid contributions of the coherence spike and vibration coherence at earlier delay times. (d) Experimental decay rates $\left(1 / \tau_{2}\right)$ obtained from global fitting of the transient absorption data and its dependence on $R$. Single-exponential fitting was performed assuming a constant offset (dashed horizontal line) obtained from the average lifetimes $\tau_{2}$ (see Table S3) of nonquenched derivatives 25 and 26. (e,f) Solvatochromic spirocyclization properties: (e) Normalized absorbance at $\lambda_{\max }$ (zwitterionic form) of 7$\mathrm{CO}_{2} \mathrm{H}$ tetrazine dyes and respective cycloadducts as a function of the dielectric constant of water/dioxane mixtures (v/v; 10/90 to 90/10). (f) Normalized absorbance at $\lambda_{\max }$ (measured in triplicates) of 7 $\mathrm{CH}_{2} \mathrm{OH}$ tetrazine dye sb-HD656 and respective cycloadduct 29 as a function of $\mathrm{pH}$.
$11.36( \pm 0.30)$ ps and $23.95( \pm 1.30)$ ps, respectively. 25 and 26 , lacking the quenching group, yielded time constants of $258( \pm 10)$ ps and $196( \pm 3)$ ps (Table S3). The interchromophore distance-dependence of the decay rate $\tau_{2}{ }^{-1}$ (Figure 2d) for $o / m / p$-TzR could be well described with an exponential fit using an offset from the unquenched reference dyes $\mathbf{2 5}$ and $\mathbf{2 6}$. These findings rule out resonance energy transfer (RET) $\left(\tau_{2}^{-1}\right.$ $\propto R^{-6}$ ) and point toward an electron-transfer-based quenching mechanism, like Dexter-type electron exchange or photoinduced electron transfer (both $\left.\tau_{2}^{-1} \propto e^{-R}\right) .^{30}$ Additionally, the spectral features of the TA signals of $o / m / p$-TzR serve to further differentiate the mechanism (see Note S2). Essentially, for $\boldsymbol{o}$-TzR, GSB, SE, and excited-state absorption (ESA) signals are recovered simultaneously, which we interpret as an indication for a solely Dexter-type electron exchange mechanism. In contrast, for $\boldsymbol{m} / \boldsymbol{p}$-TzR, the GSB/ESA recovery is delayed compared to the SE recovery. Therefore, we conclude that at these higher interchromophore distances, another quenching mechanism begins to contribute.

In $\mathrm{TA}$ measurements, we observed that fluorescence quenching occurs from an ortho-tetrazine substituent with high efficiency, yielding a low $\Phi_{\mathrm{F}}$ and a fast decay time for $\boldsymbol{o}$ TzR. This led to a high fluorescence turn-on of 95-fold in kinetic measurements upon reaction with BCN (Table 1, Figure S4). Importantly, the red-shifted analogue $\boldsymbol{o}$-TzSiR exhibited comparable fluorogenicity with a $\Phi_{\mathrm{F}}$ of $0.7 \%$ and $45-$ fold turn-on. Likewise, $\mathrm{CO}_{2} \mathrm{H}$-substituted HDyes showed turnons of 123-, 45-, 50-, and 33-fold for HD555, HD561x, HD653, and HD654x, respectively (Table 1, Figures S5-7), with the expected fast reaction kinetics upon $\mathrm{DA}_{\text {inv }}$ product formation (Figure S8).

With regards to live-cell labeling of intracellular targets, we examined the solvatochromic spirocyclization behavior that determines the cell permeability of TMR and SiR dyes. As mentioned above, 3,7-substituted HD555 and HD653 can transiently form an uncharged and nonchromophoric spirolactone from the zwitterionic and chromophoric open form. Therefore, we studied the solvent-polarity-dependent spirolactone-zwitterion equilibrium of the $7-\mathrm{CO}_{2} \mathrm{H}$ dyes. We measured the absorbance of HD555 and HD653 along with their respective cycloadducts $\mathbf{2 7}$ and $\mathbf{2 8}$ in dioxane-water mixtures of varying composition (Figure 2e, Figure S11) to determine $D_{0.5}$, the dielectric constant at half-maximum absorption ${ }^{25,31}$ (Table 1$)$. While HD555 $\left(D_{0.5} \approx 17\right)$ resided predominantly in the zwitterionic form even in low-polarity media, HD653 $\left(D_{0.5} \approx 60\right)$ had a distinct propensity to adopt to the colorless spirolactone, which is in good accordance with reports for other TMR and SiR fluorophores. ${ }^{31,32}$ Of note, 27 $\left(\mathrm{D}_{0.5} \approx 19\right)$ and $28\left(\mathrm{D}_{0.5} \approx 63\right)$ had slightly higher propensities to adopt to lactone forms than their respective tetrazine parent dyes. The formation of a nonchromophoric, spirocyclic species is also a central feature of self-blinking hydroxymethylsubstituted SiR dyes. It was shown that the reversible transition from a fluorescent zwitterion to a nonfluorescent spiroether results in fluorophore blinking that enables stochastic, switching-based super-resolution microscopy such as SMLM $^{33}$ or SOFI. ${ }^{34}$ Recently, we showed that the combination of fluorogenic labeling with self-blinking $(\mathrm{sb})$ dyes decreases background localizations in SMLM, ${ }^{35}$ and environment-sensitive sb-tetrazine probes were used for longterm SMLM. ${ }^{36}$ Our set of HDyes includes a self-blinking SiR, sb-HD656, which was synthesized from 7-hydroxy-phthalide via route 1 (Schemes $1, \mathrm{~S} 4)$. We evaluated the $\mathrm{pH}$-dependence 
Table 1. Spectral Properties of HDyes ${ }^{a}$

\begin{tabular}{|c|c|c|c|c|c|c|c|c|c|c|c|}
\hline \multirow{3}{*}{$\begin{array}{c}\text { name } \\
o \text {-TzR (26) }\end{array}$} & \multirow[b]{2}{*}{$\mathrm{X}$} & \multirow[b]{2}{*}{$\mathrm{R}^{1}$} & \multirow[b]{2}{*}{$\mathrm{R}^{2}$} & \multirow[b]{2}{*}{$\lambda_{\text {abs }}[\mathrm{nm}]$} & \multirow[b]{2}{*}{$\lambda_{\mathrm{em}}[\mathrm{nm}]$} & \multirow[b]{2}{*}{$\varepsilon\left[10^{4}(\mathrm{M} \mathrm{cm})^{-1}\right]$} & \multirow[b]{2}{*}{$\Phi_{\mathrm{F}}$} & \multirow[b]{2}{*}{$\mathrm{D}_{0.5}$} & \multirow[b]{2}{*}{ turn-on } & intra- & extra- \\
\hline & & & & & & & & & & \multicolumn{2}{|c|}{$\begin{array}{l}\text { cellular } \\
\text { application }\end{array}$} \\
\hline & $\mathrm{O}$ & $\mathrm{H}$ & $\mathrm{H}$ & $\begin{array}{l}557 \\
(557)\end{array}$ & $\begin{array}{l}583 \\
(582)\end{array}$ & $\begin{array}{l}5.52 \\
(7.41)\end{array}$ & $\begin{array}{l}0.003 \\
(0.447)\end{array}$ & - & 95 & - & - \\
\hline$o-\mathrm{TzSiR}$ & $\mathrm{SiMe}_{2}$ & $\mathrm{H}$ & $\mathrm{H}$ & 652 & 671 & 2.16 & 0.007 & - & 45 & - & - \\
\hline HD555 (27) & $\mathrm{O}$ & $\mathrm{H}$ & $\mathrm{CO}_{2} \mathrm{H}$ & $\begin{array}{l}555 \\
(556)\end{array}$ & $\begin{array}{l}581 \\
(583)\end{array}$ & $\begin{array}{l}5.10 \\
(10.0)\end{array}$ & $\begin{array}{l}0.004 \\
(0.571)\end{array}$ & $\begin{array}{l}17 \\
(19)\end{array}$ & $113 / 123^{b}$ & + & + \\
\hline HD5Slx & $\mathrm{O}$ & $\mathrm{CO}_{2} \mathrm{H}$ & $\mathrm{H}$ & 561 & 588 & 7.70 & 0.007 & - & 45 & - & + \\
\hline HD653 (28) & $\mathrm{SiMe}_{2}$ & $\mathrm{H}$ & $\mathrm{CO}_{2} \mathrm{H}$ & $\begin{array}{l}653^{d} \\
\left(652^{d}\right)\end{array}$ & $\begin{array}{l}676^{d} \\
\left(674^{d}\right)\end{array}$ & $\begin{array}{l}4.86^{d} \\
\left(5.92^{d}\right)\end{array}$ & $\begin{array}{l}0.022^{d} \\
\left(0.477^{d}\right)\end{array}$ & $\begin{array}{l}60 \\
(63)\end{array}$ & $50^{b}$ & + & + \\
\hline HD654x & $\mathrm{SiMe}_{2}$ & $\mathrm{CO}_{2} \mathrm{H}$ & $\mathrm{H}$ & 654 & 673 & 12.5 & 0.007 & - & 33 & - & + \\
\hline sb-HD656 (29) & $\mathrm{SiMe}_{2}$ & $\mathrm{H}$ & $\mathrm{CH}_{2} \mathrm{OH}$ & $\begin{array}{l}656^{c} \\
\left(656^{c}\right)\end{array}$ & $\begin{array}{l}676^{c} \\
\left(677^{c}\right)\end{array}$ & $\begin{array}{l}11.4^{c} \\
\left(10.4^{c}\right)\end{array}$ & $\begin{array}{l}0.026^{c} \\
\left(0.310^{c}\right)\end{array}$ & - & $9.0^{c}$ & + & + \\
\hline
\end{tabular}

${ }^{a}$ All measurements were performed in phosphate-buffered saline (PBS pH 7.4) and turn-on experiments with 15 eq BCN unless noted otherwise. Properties of respective BCN cycloadducts of the dyes listed in parentheses (see Figure S9 for all structures and Figure S10 for spectra). The assignment of the substituents refers to the general structure in Figure 1a. ${ }^{b} 10$ eq of EGFP-HaloTag-BCN. ${ }^{c}$ Sodium phosphate buffer $\mathrm{pH} 3.5$. ${ }^{d} 50$ mM SDS in PBS pH 7.4.
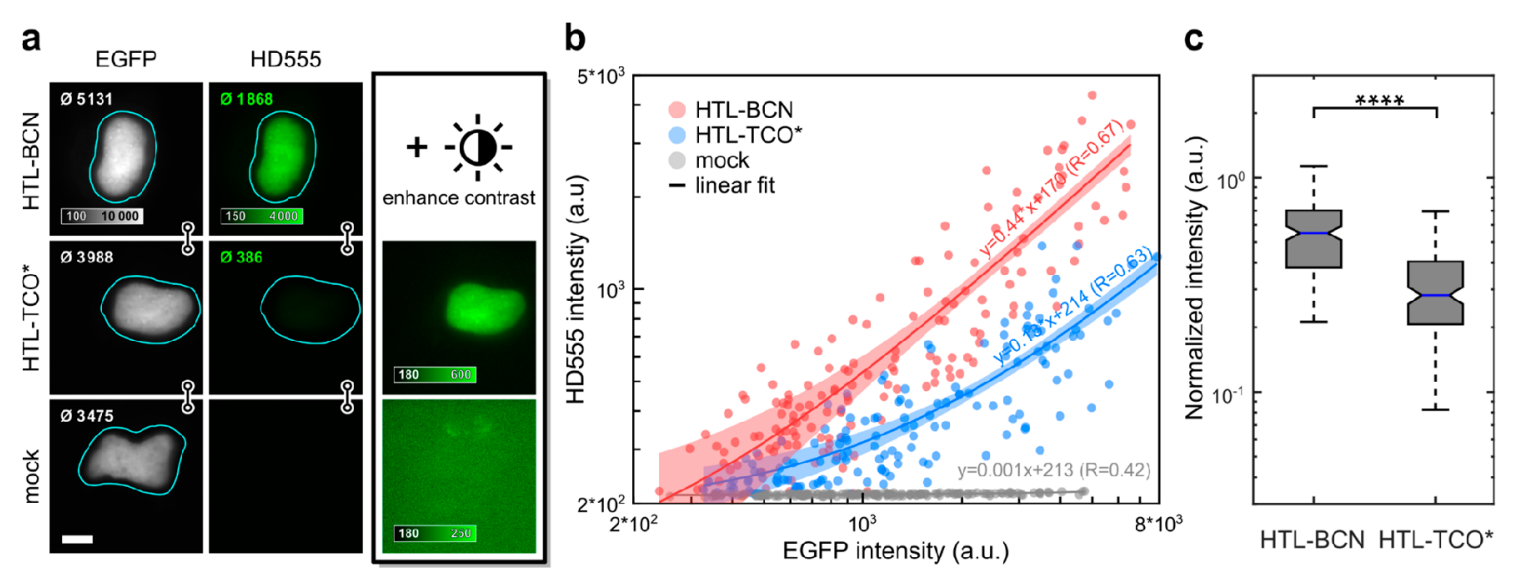

Figure 3. Fluorescence intensity quantification of HD555 in live cells. COS-7 cells expressing H2B-EGFP-HaloTag labeled with HD555 via HTL-BCN or HTL-TCO* and a mock control omitting any HaloTag linker. (a) Representative epifluorescence microscopy images of cells with comparable expression levels as indicated by mean intensities in segmented nuclei (top left corner of each image). The HD555 channel is shown at identical display settings (indicated by linked images) and with enhanced contrast. (b) Mean nucleus intensities of 151 (HTL-BCN), 145 (HTLTCO*), and 147 (mock) cells pooled from three independent experiments in EGFP and the HD555 channel. Linear regression (line) with 95\% confidence intervals. R: Kendall's tau correlation coefficient. (c) Box plot of HD555 intensities normalized to EGFP are significantly higher for HTL-BCN compared to HTL-TCO* (Wilcoxon rank-sum test, $p<10^{-23}$ ).

of the spiroether-zwitterion equilibrium of sb-HD656 and its cycloadduct 29 (Figures 2f, S12). In both cases, we observed low absorbance at physiological $\mathrm{pH}$, which indicates the predominance of the closed, nonfluorescent spiroether isomer ( $\mathrm{p} K_{\text {cycl }} 5.2$ and 5.5 for sb-HD656 and 29, respectively). The on-fraction of $\sim 5 \%$ at physiological $\mathrm{pH}$ opens the possibility of SOFI, which does not rely on single-emitter localization and is therefore compatible with a wide range of illumination and blinking conditions. ${ }^{37}$

Live-Cell Bioconjugation - Influence of Dienophiles on Fluorescence Intensities. Having confirmed generally favorable spectral and physicochemical properties of the dyes, we next evaluated their suitability for bioconjugation. Unreacted excess of the fluorophore in bioconjugation experiments is particularly difficult to remove in live-cell and in vivo experiments, leading to background signal and undesired artifacts. Here, fluorogenic probes can unfold their full potential given they maintain fluorogenicity in live cells. Therefore, after having shown successful in vitro labeling of BCN-modified EGFP (Figure S13), we evaluated the fluorogenicity of HDyes in live-cell labeling and imaging. While we used BCN as a dienophile for in vitro characterization due to its formation of a single product, we tested both, $\mathrm{BCN}$ and trans-cyclooctene (TCO), as dienophiles in live cells. TCO derivatives have been reported to possess superior kinetics over $\mathrm{BCN},{ }^{38-40}$ and it is further known that the choice of the $\mathrm{DA}_{\text {inv }}$ dienophile can influence the fluorescence turn-on of tetrazine dyes. ${ }^{26,41,42}$ We therefore compared the brightness of cell-permeable HD555 upon conjugation with a BCN- or (E)-cyclooct-2-en-1-ol (TCO*)-modified intracellular target. First, COS-7 cells transiently expressing the histone H2B fusion protein $\mathrm{H} 2 \mathrm{~B}-\mathrm{EGFP}-\mathrm{HaloTag}$ were loaded with respective HaloTag ligand-dienophile (HTL-dienophile), a bifunctional linker, to provide specific binding sites for fluorogenic labeling with HD555. With HTL-BCN, we observed specific staining with excellent contrast after dye incubation at $1 \mu \mathrm{M}$ over $2 \mathrm{~h}$ and mere buffer replacement (Figure 3a, top row). Second, we tested $\mathrm{TCO}^{*}$ as a $\mathrm{DA}_{\text {inv }}$ dienophile under identical conditions. Here, we observed a specific signal but substantially lower contrast (Figure 3a, 

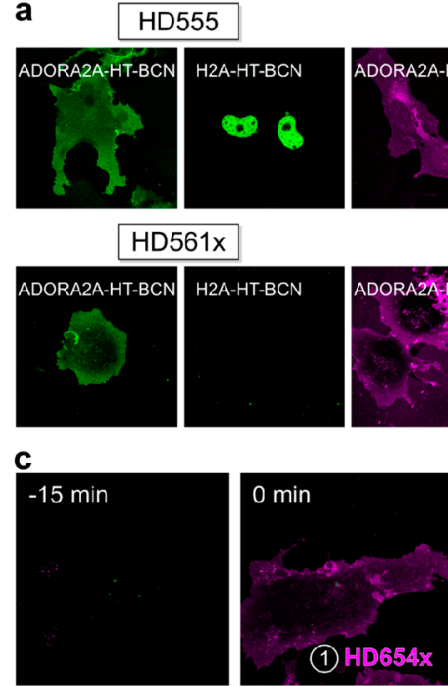

d
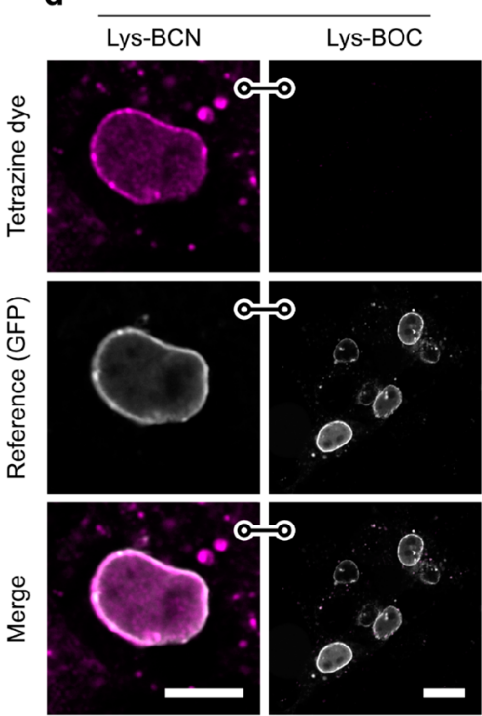
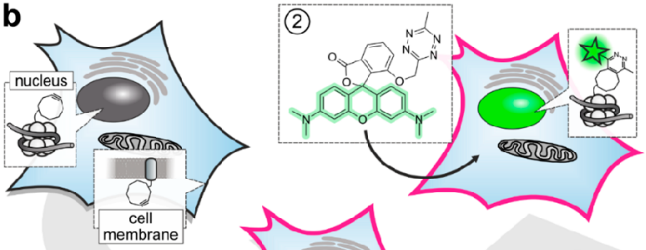

HD654x
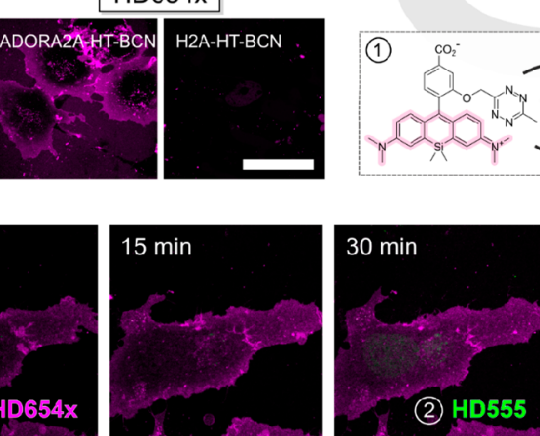

$\mathrm{SiR}-\mathrm{Tz}$
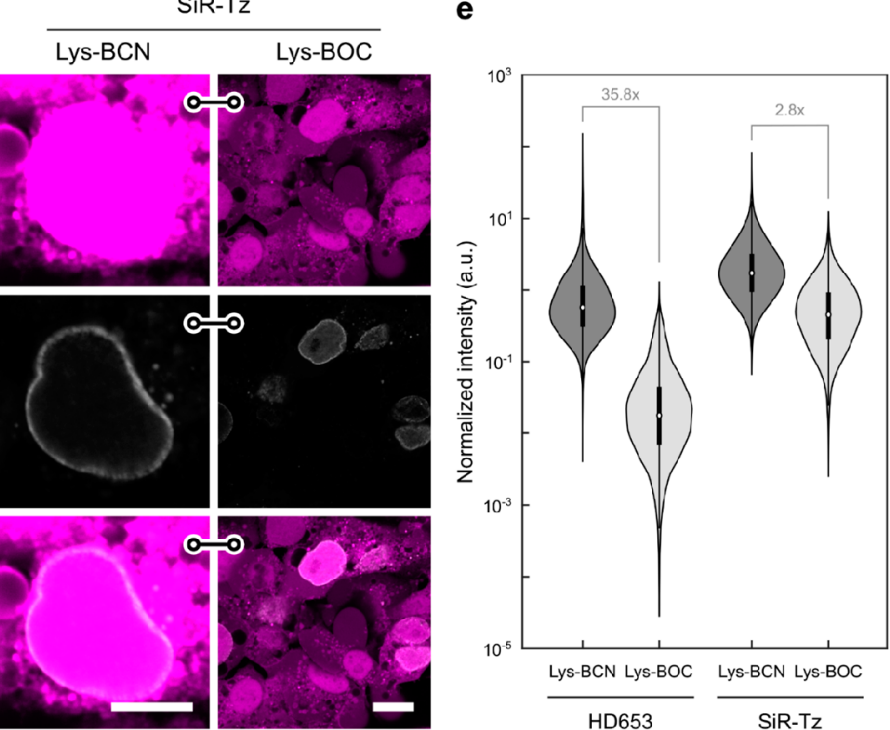

Figure 4. Wash-free multicolor and unnatural amino acid imaging in live cells. (a) Wash-free labeling of intra- and extracellular targets in COS-7 cells. Cells were transiently transfected with ADORA2A-HaloTag (plasma membrane) or H2A-HaloTag (nucleus), loaded with HTL-BCN, and incubated with HDyes at $1 \mu \mathrm{M}$ for $30 \mathrm{~min}$. Scale bar $50 \mu \mathrm{m}$. For reference and controls, see Figures S16-19. (b) Dual-color labeling with a single chemistry. Intra- and extracellular proteins $\mathrm{H} 2 \mathrm{~A}$ and ADORA2A are targeted via HaloTag and $\mathrm{HTL}-\mathrm{BCN}$ and then incubated with cellimpermeable HD654x for 30 min followed by addition of HD555. (c) Time-lapse confocal microscopy shows labeling of intra- and extracellular targets with spectrally distinct fluorophores can be achieved without any washing. Scale bar $20 \mu \mathrm{m}$. For individual color channels and reference staining, see (Figure S23). (d) Un-natural amino acid labeling of PEGFP $^{\mathrm{N149TAG}}-\mathrm{Nup} 153$ with Lys-BCN or Lys-BOC (control) in COS-7 cells. Live-cell confocal microscopy after incubation with tetrazine dyes $\mathbf{H D 6 5 3}$ and SiR-Tz at $500 \mathrm{nM}$ for 30 min and mere buffer replacement. Scale bars: $10 \mu \mathrm{m}$ (Lys-BCN) or $20 \mu \mathrm{m}$ (Lys-BOC). (e) Quantitative comparison of target and background labeling with flow cytometry analysis. pEGFP $^{\text {N149TAG }}-$ Nup153 COS-7 cells were loaded with either Lys-BCN for specific or Lys-BOC for unspecific labeling. Intensity of HD653 and SiR-Tz was normalized to EGFP and signal-to-background ratios calculated from population medians.

middle row). Accordingly, mean intensities (see inset in nuclei images) of the nucleus were significantly lower for HTLTCO* compared to HTL-BCN. A mock control omitting HTL-dienophiles (Figure $3 \mathrm{a}$, bottom row) showed no unspecific staining from unreacted dye with intensity values at background level. For a quantitative evaluation of fluorescence intensities with $\mathrm{HTL}-\mathrm{BCN}$ or HTL-TCO*, we compared intranuclear HD555 intensities against EGFP intensity, which served as an expression control (Figure 3b). For both dienophiles, we observed a linear correlation of HD555 and EGFP signal despite considerable cell-to-cell variation (Figure S14) and a 3.4-fold higher intensity obtained using HTL-BCN compared to HTL-TCO*. Considering the observed variability, we additionally compared HD555 intensities normalized to EGFP, confirming the significantly reduced emission intensity obtained with $\mathrm{TCO}^{*}$ in live cells (Figure 3c).

This was in good accordance with the different emission intensities obtained after reaction of $\mathbf{H D 5 5 5}$ with $\mathrm{BCN}$ and $\mathrm{TCO}^{*}$ in vitro (Figure S15) and recent work by others that reported a strong dependency of fluorescence enhancement in $\mathrm{DA}_{\text {inv }}$ on the used dienophile. ${ }^{42,43}$ Due to the higher obtainable fluorescence brightness, we employed $\mathrm{BCN}$ as a $\mathrm{DA}_{\mathrm{inv}}$ dienophile for cellular labeling in all following experiments.

Intra- and Extracellular Wash-Free Protein Imaging in Living Cells. Cell permeability of synthetic small-molecule 
a

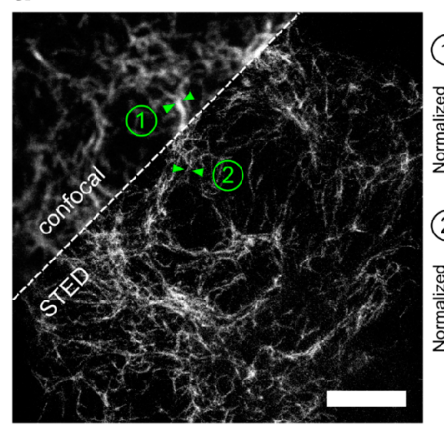

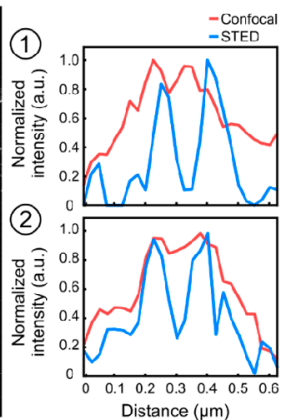

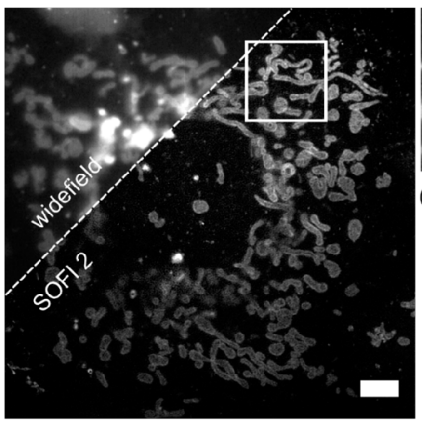

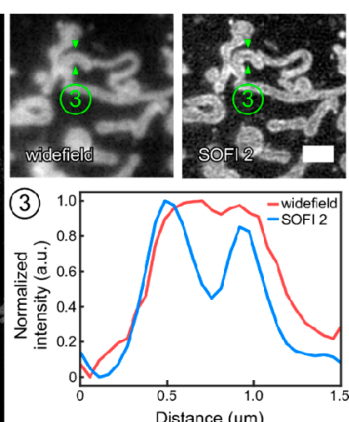

Distance $(\mu \mathrm{m})$

C
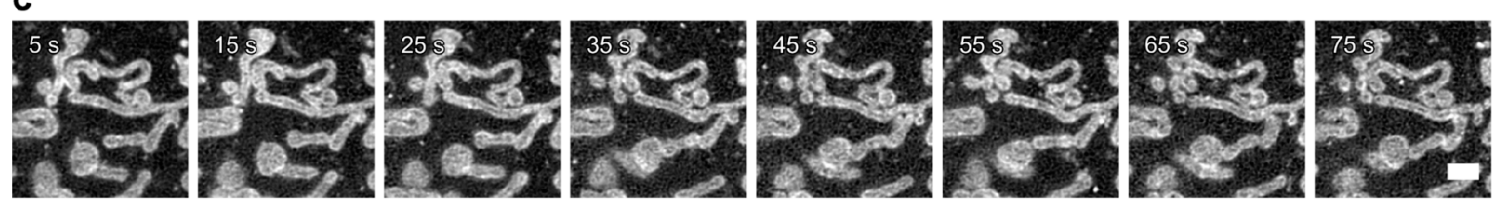

d
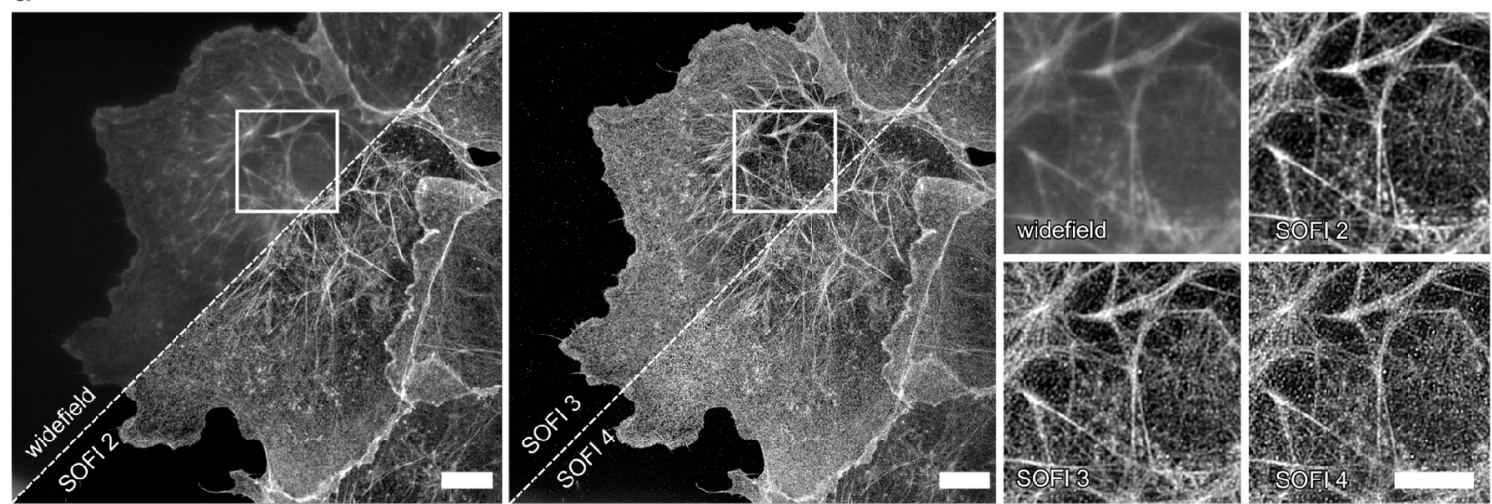

Figure 5. Super-resolution microscopy with HDyes. (a) Live-cell confocal and STED microscopy of COS-7 cells expressing pVimentin ${ }^{\text {N116TAG } \rightarrow B C N}$ labeled with $\mathrm{HD} 653$ with intensity line profiles showing the resolution improvement. Scale bar $5 \mu \mathrm{m}$. (b) Live-cell widefield (temporal average of series) and second-order SOFI of mitochondria in COS-7 cells transiently expressing TOMM20-mCherry-HaloTag labeled with sb-HD656 via HTL-BCN. Scale bar $5 \mu \mathrm{m}$. The close-ups correspond to the ROI indicated in the overview image. The cross-sectional profile of the averaged widefield image and second-order SOFI shows the improved image contrast and resolution. Scale bar $2 \mu \mathrm{m}$. (c) Time course of second-order SOFI corresponding to the ROI indicated in (b) showing the movement of mitochondria with a time resolution of $10 \mathrm{~s}$ (see Figure S28 for the full sequence). Image acquisition: 500 frames per time point, $20 \mathrm{~ms}$ exposure time, $635 \mathrm{~nm}$ laser, $140 \mathrm{~W} / \mathrm{cm}^{2}$. The images are representative of more than five cells from two independent experiments. (d) High-order SOFI imaging of sb-HD656 labeled f-actin in fixed COS-7 cells showing SOFI analysis up to fourth cumulant order. Image acquisition: 20000 frames, $50 \mathrm{~ms}$ exposure time, $635 \mathrm{~nm}$ laser, $275 \mathrm{~W} / \mathrm{cm}^{2}$. The images are representative of six cells from two independent experiments. Scale bars $10 \mu \mathrm{m}$.

fluorophores is a crucial factor in live-cell imaging. Hence, after having identified a suitable $\mathrm{DA}_{\text {inv }}$ dienophile for live-cell conditions, we next evaluated the cell permeability of the newly developed tetrazine dye series, including the potentially cellimpermeable 3,5-substituted derivatives. This was tested using HTL-BCN conjugated to HaloTag fusion proteins with intraor extracellular localization. In transiently transfected COS-7 cells, all four HDyes showed specific extracellular labeling of adenosine $A_{2 A}$ receptor (ADORA2A) via HaloTag-BCN. Incubation with $1 \mu \mathrm{M}$ dye for $30 \mathrm{~min}$ without further washing yielded uniform membrane staining with negligible background and excellent overlap with a reference label (Figures 4a, S16-19). To address an intracellular target, we incubated COS-7 cells transiently expressing histone H2A-HaloTag loaded with $\mathrm{HTL}-\mathrm{BCN}$ for $30 \mathrm{~min}$ with $1 \mu \mathrm{M}$ dye. Imaging under wash-free conditions showed that only HD555 and HD653, dyes capable of forming transient spirolactones, were able to pass through the plasma membrane and specifically label the nuclear target. Using HD654x and HD561x under identical labeling and imaging conditions showed no nuclear signal (Figures 4a, S16-19). Controls without HTL-BCN or HaloTag fusion proteins yielded negligible signals for all four dyes (Figures S16-19, S22). In contrast to these zwitterionic and net neutral dyes, the positively charged dyes $\boldsymbol{o}$-TzR and $\boldsymbol{o}$ TzSiR showed significant unspecific mitochondrial accumulation (Figures S20, S21). To confirm the improved fluorogenicity and live-cell applicability of the new red-emitting dyes, we compared the SiR derivative HD653 with previously reported tetrazine-SiR conjugates in live-cell imaging. Under no-wash conditions, HD653 showed highly specific labeling and no visible off-target staining, while all other $\mathrm{SiR}$ derivatives tested exhibited varying degrees of off-target staining (Figure S22).

We then used complementary dye pairs with distinct cell permeability and absorption/emission wavelengths to perform dual-target labeling for two-color microscopy. Frist, we labeled an extracellular target at saturating concentrations with cellimpermeable HD654x, followed by labeling of an intracellular 
target with cell-permeable HD555 (Figure 4b). This concept allows to exploit the high efficiency of labeling via $\mathrm{DA}_{\text {inv }}$ with fluorogenic tetrazines to consecutively label two targets and overcomes the need for orthogonal labeling reactions. To demonstrate this, we coexpressed ADORA2A-HaloTag and $\mathrm{H} 2 \mathrm{~A}-\mathrm{HaloT}$ ag in COS7 cells and treated the cells with HTL$\mathrm{BCN}$. After washing to remove excess $\mathrm{HTL}-\mathrm{BCN}$, we incubated the cells with HD654x at $1 \mu \mathrm{M}$ for $30 \mathrm{~min}$, followed by a second 30 min incubation with HD555 at $1 \mu \mathrm{M}$. Image acquisition was performed over time without additional washing steps after incubation with HDyes (Figure 4c). To achieve orthogonal labeling, complete saturation of extracellular BCN-modified proteins with the impermeable dye is crucial, to avoid cross reactivity of the second dye. This is achievable due to high reaction rates of the $\mathrm{DA}_{\mathrm{inv}}$ reaction and elevated dye concentrations facilitated by effective quenching of unreacted dyes. Accordingly, unspecific background and cross-labeling of remaining extracellular target with the second added dye was not observed (for individual color channel images, see Figure S23). After the stepwise incubation with HD655x and HD555 for a total of $60 \mathrm{~min}$ without further washing, we observed high contrast specific target labeling (Figure 4c), which could be confirmed by reference chase staining with HTL-Rhodamine110 (Figure S23). Spectrally interchanged labeling using HD561x for extra- and HD653 for intracellular labeling yielded the expected comparable results (Figure S24). Therefore, our dyes enable live-cell two-color microscopy of an intra- and extracellular target under wash-free conditions with full spectral flexibility.

Encouraged by these results for intra- and extracellular labeling using protein tags, we next moved on to apply HDyes for live-cell protein labeling using UAAs. The labeling of UAAmodified proteins with small-molecule fluorophores via $\mathrm{DA}_{\mathrm{inv}}$ results in a minimal size modification, which reduces unwanted perturbation of the target protein. ${ }^{28,44}$ Due to low cellular autofluorescence in the far-red spectral region, we anticipated the highest contrast from SiR dyes. We therefore used HD653 for site-specific labeling of the nuclear pore complex via the Amber (TAG) mutant nucleoporin 153 construct $\left(\right.$ EGFP $\left.^{\mathrm{N} 149 T A G}-\mathrm{Nup} 153\right)$ and performed a side-by-side comparison with commercial SiR-Tz (for structure, see Figure S25). To account for an additional fluorogenic effect due to environmental influence on the zwitterion-spirolactone equilibrium, we determined fluorescence turn-on values with a purified BCN-tagged protein of 55-fold for HD653 and 3.1fold for SiR-Tz (Figure S7). COS-7 cells were transiently transfected with the Nup153 construct in the presence of the respective genetic code expansion machinery and Lys-BCN, washed, and then labeled with HD653 or SiR-Tz. Confocal microscopy (Figure 4d) showed that HD653 yields comparable specific signal but substantially lower off-target background than SiR-Tz. We then used nonreactive LysBOC to further assess the labeling specificity and observed unspecific background signal from SiR-Tz in contrast to barely detectable background from HD653 (Figure 4d). To quantify the labeling specificity, we performed flow cytometry analysis of cells expressing the NUP153 construct in presence (Lys$\mathrm{BCN}$ ) or absence (Lys-BOC) of specific reaction sites for HD653 or SiR-Tz, respectively (Figures 4e and S26). Due to efficient quenching, the difference in signal of HD653 $(35.8 \times)$ was substantially higher compared to $\mathrm{SiR}-\mathbf{T z}(2.8 \times)$. This shows that the high fluorogenicity of $\mathrm{HD} 653$ allows for UAA live-cell labeling with minimal washing and enables high signalto-background ratio for fluorescence imaging applications.

STED and SOFI Super-resolution Microscopy. With improving spatial resolution of imaging techniques, the size of fluorescent labels becomes increasingly important. Having shown that specific labeling of UAA with improved contrast could be achieved with our dyes, we evaluated their use in super-resolution microscopy. In this context, we used HD653 in combination with UAAs as minimal size labels. Based on previous reports using structurally akin SiRs, ${ }^{12,24,32}$ we evaluated the performance of HD653 in STED microscopy. To test its applicability, we labeled the Amber mutant vimentin $^{\mathrm{N} 116 \mathrm{TAG}}-\mathrm{mOrange}$ construct with $\mathrm{Lys}-\mathrm{BCN}$ and HD653 in live COS-7 cells. STED imaging using a $775 \mathrm{~nm}$ continuous wave laser for depletion resolved vimentin structures and clearly showed enhanced resolution compared to confocal microscopy (Figure 5a). Of note, after 50 cycles of STED imaging, bleaching for HD653-labeled vimentin was lower compared to commercial SiR-Tz (Figure S27).

Another approach for super-resolution microscopy uses stochastic temporal emission fluctuations of single fluorophores. Based on our own previous work, ${ }^{35}$ we used a selfblinking fluorogenic tetrazine-SiR for minimally invasive livecell super-resolution microscopy. While SMLM can achieve resolutions down to a few nanometers, ${ }^{45}$ it requires sparse signals to allow for localization of individual emitters. In contrast, SOFI exploits spatially and temporally correlated intensity fluctuations for resolution improvement without the need of isolated emitters. ${ }^{46}$ This is especially beneficial for livecell microscopy of highly abundant targets at low excitation intensities. We used the self-blinking derivative sb-HD656 in combination with SOFI for subdiffraction imaging in living and fixed cells. First, we performed live-cell imaging of mitochondrial import receptor protein TOMM20 labeled via HaloTag and HTL-BCN with sb-HD656 at low illumination intensities in the near-infrared $\left(\sim 140 \mathrm{~W} / \mathrm{cm}^{2}\right.$ at $\left.635 \mathrm{~nm}\right)$. Due to low phototoxicity, it was possible to perform time-lapse imaging of the rearrangement of the mitochondrial network in COS-7 cells (Figure 5c, for longer time course see Figure S28). Second-order cumulant analysis required only a few hundred frames and resolved the mitochondrial outer membrane (see close-up and line profiles in Figure 5b). In contrast to SMLM, SOFI was also compatible with higher active emitter densities as encountered with highly abundant histone protein $\mathrm{H} 2 \mathrm{~B}$ in nuclei of live COS-7 cells (Figure S29).

In order to demonstrate higher-order SOFI imaging enabled by the high photostability of HDyes, sb-HD656 was used to stain the cytoskeletal actin network. We exploited the versatility offered by $\mathrm{DA}_{\text {inv }}$ tetrazine labeling and used a small-molecule labeling approach with phalloidin, a natural toxin with a high affinity to f-actin to introduce $\mathrm{BCN}$ as $\mathrm{DA}_{\text {inv }}$ reaction partner. To this end, we targeted f-actin with phalloidin-BCN in fixed COS-7 cells and subsequently labeled it with sb-HD656. The $\mathrm{pH}$ of the imaging buffer was chosen for a reduced on/off ratio for high-order SOFI analysis (Figure S30). sb-HD656 was found to be photostable at relatively low illumination intensities $\left(275 \mathrm{~W} / \mathrm{cm}^{2}\right)$, thus allowing the acquisition of up to 20000 frames with moderately low photobleaching (Figure S31). SOFI analysis was performed up to fourth cumulant order while maintaining sufficiently high signal-to-noise ratio (Figure 5d). Compared to widefield imaging, second-order SOFI provided a significant contrast enhancement, while higher-order (third-fourth) SOFI images 
allowed to resolve individual actin filaments that were not distinguishable in widefield images. Additionally, we compared sb-HD656 to a previously published self-blinking tetrazine probe. $^{35}$ The use of sb-HD656 resulted in higher on/off ratios and on times which makes it well suited for SOFI (Figure S32).

\section{CONCLUSION}

We developed a series of red and far-red fluorogenic tetrazine dyes (HDyes) for live-cell bio-orthogonal labeling of intra- and extracellular targets. The design rationale with tetrazines flexibly linked to the fluorophore at minimal distance led to efficient fluorescence quenching by Dexter exchange. HDyes exhibited high fluorogenicity and were well-suited for live-cell imaging under wash-free conditions as demonstrated in twocolor labeling experiments and STED super-resolution imaging of UAA-labeled target proteins. The modular synthetic route provided a self-blinking HDye, which was successfully exploited in long-term live-cell SOFI imaging. We expect the HDyes to leverage applications of bio-orthogonal chemistry in the field of super-resolution microscopy. Their excellent photophysical properties, improved signal-to-background ratios, and small labeling size are ideal prerequisites for the direct labeling of UAA-modified proteins, which has great potential of reducing the linkage error. We expect the herein presented tetrazine-xanthene scaffold to serve as general motif in the development of numerous other fluorogenic probes in the future.

\section{ASSOCIATED CONTENT}

\section{SI Supporting Information}

The Supporting Information is available free of charge at https://pubs.acs.org/doi/10.1021/acscentsci.1c00703.

Syntheses, compound characterization data, and details of the computational and time-resolved spectroscopy studies as well as of imaging experiments (PDF)

Structural data of HD555 as a Crystallographic Information File (CIF)

\section{AUTHOR INFORMATION}

\section{Corresponding Authors}

Dirk-Peter Herten - Institute of Physical Chemistry, Heidelberg University, 69120 Heidelberg, Germany; Centre of Membrane Proteins and Receptors (COMPARE), Universities of Birmingham and Nottingham, B15 2TT Birmingham, U.K.; College of Medical and Dental Sciences, Medical School \& School of Chemistry, University of Birmingham, B15 2TT Birmingham, U.K.; Email: d.herten@bham.ac.uk

Richard Wombacher - Institute of Pharmacy and Molecular Biotechnology, Heidelberg University, 69120 Heidelberg, Germany; Department of Chemical Biology, Max Planck Institute for Medical Research, 69120 Heidelberg, Germany; ○ orcid.org/0000-0002-3027-7778; Email: wombacher@ mr.mpg.de

\section{Authors}

Philipp Werther - Institute of Pharmacy and Molecular Biotechnology, Heidelberg University, 69120 Heidelberg, Germany

Klaus Yserentant - Institute of Physical Chemistry, Heidelberg University, 69120 Heidelberg, Germany; Centre of
Membrane Proteins and Receptors (COMPARE), Universities of Birmingham and Nottingham, B15 2TT Birmingham, U.K.; College of Medical and Dental Sciences, Medical School \& School of Chemistry, University of Birmingham, B15 2TT Birmingham, U.K.; Faculty of Biosciences, Heidelberg University, 69120 Heidelberg, Germany

Felix Braun - Institute of Physical Chemistry, Heidelberg University, 69120 Heidelberg, Germany

Kristin Grußmayer - Laboratory of Nanoscale Biology, Ecole Polytechnique Fédérale de Lausanne (EPFL), CH-1015 Lausanne, Switzerland; Delft University of Technology, Kavli Institute of Nanoscience, Department of Bionanoscience, 2629 HZ Delft, The Netherlands

Vytautas Navikas - Laboratory of Nanoscale Biology, Ecole Polytechnique Fédérale de Lausanne (EPFL), CH-1015 Lausanne, Switzerland

Miao Yu - Biocenter Mainz, Departments of Biology and Chemistry, Johannes Gutenberg University, 55128 Mainz, Germany; Institute of Molecular Biology, 55128 Mainz, Germany; Structural and Computational Biology Unit, Cell Biology and Biophysics Unit, EMBL, 69117 Heidelberg, Germany

Zhibin Zhang - Institute of Physical Chemistry, Heidelberg University, 69120 Heidelberg, Germany; National Key Laboratory of Science and Technology on Tunable Laser, Harbin Institute of Technology, Harbin 150080, China

Michael J. Ziegler - Institute of Pharmacy and Molecular Biotechnology, Heidelberg University, 69120 Heidelberg, Germany; Department of Chemical Biology, Max Planck Institute for Medical Research, 69120 Heidelberg, Germany

Christoph Mayer - Institute of Pharmacy and Molecular Biotechnology, Heidelberg University, 69120 Heidelberg, Germany

Antoni J. Gralak - Institute of Pharmacy and Molecular Biotechnology, Heidelberg University, 69120 Heidelberg, Germany

Marvin Busch - Institute of Pharmacy and Molecular Biotechnology, Heidelberg University, 69120 Heidelberg, Germany

Weijie Chi - Fluorescence Research Group, Singapore University of Technology and Design, 487372, Singapore; (1) orcid.org/0000-0003-1776-0025

Frank Rominger - Institute of Organic Chemistry, Heidelberg University, 69120 Heidelberg, Germany

Aleksandra Radenovic - Laboratory of Nanoscale Biology, Ecole Polytechnique Fédérale de Lausanne (EPFL), CH-1015 Lausanne, Switzerland; 이이.org/0000-0001-8194-2785

Xiaogang Liu - Fluorescence Research Group, Singapore University of Technology and Design, 487372, Singapore; (1) orcid.org/0000-0002-2553-2068

Edward A. Lemke - Biocenter Mainz, Departments of Biology and Chemistry, Johannes Gutenberg University, 55128 Mainz, Germany; Institute of Molecular Biology, 55128 Mainz, Germany; Structural and Computational Biology Unit, Cell Biology and Biophysics Unit, EMBL, 69117 Heidelberg, Germany

Tiago Buckup - Institute of Physical Chemistry, Heidelberg University, 69120 Heidelberg, Germany; 이이.org/00000002-1194-0837

Complete contact information is available at:

https://pubs.acs.org/10.1021/acscentsci.1c00703 


\section{Author Contributions}

\#\# PW and KY contributed equally to this work.

\section{Notes}

The authors declare no competing financial interest.

Crystallographic data has been deposited at the Cambridge Crystallographic Data Centre (CCDC) under the CCDC deposition number 2021104. The data can be obtained free of charge via https://www.ccdc.cam.ac.uk/structures.

\section{ACKNOWLEDGMENTS}

RW and EAL acknowledge funding from the Deutsche Forschungsgemeinschaft DFG (SPP1623) and DPH from the Federal Ministry of Education and Research (BMBF/VDI; MorphiQuant3D and Switch-Click-Microscopy) and the DFG (HE4559/5-1, HE4559/6-1). DPH gratefully acknowledges funding by the Centre of Membrane Proteins and Receptors (COMPARE, Universities of Birmingham and Nottingham). EAL also thanks ERC SMPFv2.0 for funding. AR, KG, and VN thank the EPFL BioImaging \& Optics Core Facility (EPFLBIOP) and Horizon 2020 research and innovation program of the European Union via grant 686271/SEFRI 16.0047 for funding. XL acknowledges funding from Singapore University of Technology and Design (T1SRCI17126). ZZ acknowledges funding from the National Natural Science Foundation of China (Grant No. 61675057) and China Scholarship Council. MJZ acknowledges a fellowship by the Carl-Zeiss-Stiftung. We thank Carina Lämmle for providing a tetrazine compound for Figure S22. We gratefully acknowledge access to the Nikon Imaging Center at Heidelberg University. We thank Daniel Wolf and Frederik Weber for experimental help and Prof. Dr. Andres Jäschke for constant support.

\section{REFERENCES}

(1) Wang, L.; Frei, M. S.; Salim, A.; Johnsson, K. Small-Molecule Fluorescent Probes for Live-Cell Super-Resolution Microscopy. J. Am. Chem. Soc. 2019, 141 (7), 2770-2781.

(2) Lang, K.; Chin, J. W. Bioorthogonal Reactions for Labeling Proteins. ACS Chem. Biol. 2014, 9 (1), 16-20.

(3) Plass, T.; Milles, S.; Koehler, C.; Schultz, C.; Lemke, E. A. Genetically Encoded Copper-Free Click Chemistry. Angew. Chem., Int. Ed. 2011, 50 (17), 3878-3881.

(4) Schoch, J.; Wiessler, M.; Jäschke, A. Post-Synthetic Modification of DNA by Inverse-Electron-Demand Diels-Alder Reaction. J. Am. Chem. Soc. 2010, 132 (26), 8846-8847.

(5) Ploschik, D.; Rönicke, F.; Beike, H.; Strasser, R.; Wagenknecht, H.-A. DNA Primer Extension with Cyclopropenylated 7-Deaza-2'Deoxyadenosine and Efficient Bioorthogonal Labeling in Vitro and in Living Cells. ChemBioChem 2018, 19 (18), 1949-1953.

(6) Agarwal, P.; Beahm, B. J.; Shieh, P.; Bertozzi, C. R. Systemic Fluorescence Imaging of Zebrafish Glycans with Bioorthogonal Chemistry. Angew. Chem., Int. Ed. 2015, 54 (39), 11504-11510.

(7) Shieh, P.; Dien, V. T.; Beahm, B. J.; Castellano, J. M.; WyssCoray, T.; Bertozzi, C. R. CalFluors: A Universal Motif for Fluorogenic Azide Probes across the Visible Spectrum. J. Am. Chem. Soc. 2015, 137 (22), 7145-7151.

(8) Yang, J.; Sečkute, J.; Cole, C. M.; Devaraj, N. K. Live-Cell Imaging of Cyclopropene Tags with Fluorogenic Tetrazine Cycloadditions. Angew. Chem., Int. Ed. 2012, 51 (30), 7476-7479.

(9) Neef, A. B.; Schultz, C. Selective Fluorescence Labeling of Lipids in Living Cells. Angew. Chem., Int. Ed. 2009, 48 (8), 1498-1500.

(10) Nadler, A.; Schultz, C. The Power of Fluorogenic Probes. Angew. Chem., Int. Ed. 2013, 52 (9), 2408-2410.

(11) Shieh, P.; Bertozzi, C. R. Design Strategies for Bioorthogonal Smart Probes. Org. Biomol. Chem. 2014, 12 (46), 9307-9320.
(12) Lukinavičius, G.; Umezawa, K.; Olivier, N.; Honigmann, A.; Yang, G.; Plass, T.; Mueller, V.; Reymond, L.; Corrêa Jr, I. R.; Luo, Z.G.; Schultz, C.; Lemke, E. A.; Heppenstall, P.; Eggeling, C.; Manley, S.; Johnsson, K. A Near-Infrared Fluorophore for Live-Cell SuperResolution Microscopy of Cellular Proteins. Nat. Chem. 2013, 5, 132-139.

(13) Grimm, J. B.; English, B. P.; Chen, J.; Slaughter, J. P.; Zhang, Z.; Revyakin, A.; Patel, R.; Macklin, J. J.; Normanno, D.; Singer, R. H.; Lionnet, T.; Lavis, L. D. A General Method to Improve Fluorophores for Live-Cell and Single-Molecule Microscopy. Nat. Methods 2015, 12, 244-250.

(14) Wieczorek, A.; Werther, P.; Euchner, J.; Wombacher, R. Greento Far-Red-Emitting Fluorogenic Tetrazine Probes-Synthetic Access and No-Wash Protein Imaging inside Living Cells. Chem. Sci. 2017, 8 (2), 1506-1510.

(15) Wang, L.; Tran, M.; D’Este, E.; Roberti, J.; Koch, B.; Xue, L.; Johnsson, K. A General Strategy to Develop Cell Permeable and Fluorogenic Probes for Multicolour Nanoscopy. Nat. Chem. 2020, 12 (2), 165-172.

(16) Devaraj, N. K.; Hilderbrand, S.; Upadhyay, R.; Mazitschek, R.; Weissleder, R. Bioorthogonal Turn-on Probes for Imaging Small Molecules inside Living Cells. Angew. Chem., Int. Ed. 2010, 49 (16), 2869-2872.

(17) Meimetis, L. G.; Carlson, J. C. T.; Giedt, R. J.; Kohler, R. H.; Weissleder, R. Ultrafluorogenic Coumarin-Tetrazine Probes for RealTime Biological Imaging. Angew. Chem., Int. Ed. 2014, 53 (29), 75317534.

(18) Carlson, J. C. T.; Meimetis, L. G.; Hilderbrand, S. A.; Weissleder, R. BODIPY-Tetrazine Derivatives as Superbright Bioorthogonal Turn-on Probes. Angew. Chem., Int. Ed. 2013, 52 (27), 6917-6920.

(19) Wieczorek, A.; Buckup, T.; Wombacher, R. Rigid Tetrazine Fluorophore Conjugates with Fluorogenic Properties in the Inverse Electron Demand Diels-Alder Reaction. Org. Biomol. Chem. 2014, 12 (24), 4177-4185.

(20) Chen, L.; Li, F.; Nandi, M.; Huang, L.; Chen, Z.; Wei, J.; Chi, W.; Liu, X.; Yang, J. Towards Tetrazine-Based near-Infrared Fluorogenic Dyes: Is There a Wavelength Limit? Dyes Pigm. 2020, $177,108313$.

(21) Lee, Y.; Cho, W.; Sung, J.; Kim, E.; Park, S. B. Monochromophoric Design Strategy for Tetrazine-Based Colorful Bioorthogonal Probes with a Single Fluorescent Core Skeleton. J. Am. Chem. Soc. 2018, 140 (3), 974-983.

(22) Heilemann, M.; van de Linde, S.; Mukherjee, A.; Sauer, M. Super-Resolution Imaging with Small Organic Fluorophores. Angew. Chem., Int. Ed. 2009, 48 (37), 6903-6908.

(23) Van De Linde, S.; Löschberger, A.; Klein, T.; Heidbreder, M.; Wolter, S.; Heilemann, M.; Sauer, M. Direct Stochastic Optical Reconstruction Microscopy with Standard Fluorescent Probes. Nat. Protoc. 2011, 6 (7), 991-1009.

(24) Lukinavičius, G.; Reymond, L.; D’Este, E.; Masharina, A.; Göttfert, F.; Ta, H.; Güther, A.; Fournier, M.; Rizzo, S.; Waldmann, H.; Blaukopf, C.; Sommer, C.; Gerlich, D. W.; Arndt, H.-D.; Hell, S. W.; Johnsson, K. Fluorogenic Probes for Live-Cell Imaging of the Cytoskeleton. Nat. Methods 2014, 11, 731-733.

(25) Lukinavičius, G.; Reymond, L.; Umezawa, K.; Sallin, O.; D’Este, E.; Göttfert, F.; Ta, H.; Hell, S. W.; Urano, Y.; Johnsson, K. Fluorogenic Probes for Multicolor Imaging in Living Cells. J. Am. Chem. Soc. 2016, 138 (30), 9365-9368.

(26) Wu, H.; Yang, J.; Šckkute, J.; Devaraj, N. K. In Situ Synthesis of Alkenyl Tetrazines for Highly Fluorogenic Bioorthogonal Live-Cell Imaging Probes. Angew. Chem., Int. Ed. 2014, 53 (23), 5805-5809.

(27) Kozma, E.; Estrada Girona, G.; Paci, G.; Lemke, E. A.; Kele, P. Bioorthogonal Double-Fluorogenic Siliconrhodamine Probes for Intracellular Super-Resolution Microscopy. Chem. Commun. 2017, 53 (50), 6696-6699.

(28) Beliu, G.; Kurz, A. J.; Kuhlemann, A. C.; Behringer-Pliess, L.; Meub, M.; Wolf, N.; Seibel, J.; Shi, Z.-D.; Schnermann, M.; Grimm, J. B.; Lavis, L. D.; Doose, S.; Sauer, M. Bioorthogonal Labeling with 
Tetrazine-Dyes for Super-Resolution Microscopy. Commun. Biol. 2019, 2 (1), 261.

(29) Grimm, J. B.; Brown, T. A.; Tkachuk, A. N.; Lavis, L. D. General Synthetic Method for Si-Fluoresceins and Si-Rhodamines. ACS Cent. Sci. 2017, 3 (9), 975-985.

(30) Lakowicz, J. R. Mechanisms and Dynamics of Fluorescence Quenching. Principles of Fluorescence Spectroscopy 2006, 331-351.

(31) Butkevich, A. N.; Mitronova, G. Y.; Sidenstein, S. C.; Klocke, J. L.; Kamin, D.; Meineke, D. N. H.; D’Este, E.; Kraemer, P.-T.; Danzl, J. G.; Belov, V. N.; Hell, S. W. Fluorescent Rhodamines and Fluorogenic Carbopyronines for Super-Resolution STED Microscopy in Living Cells. Angew. Chem., Int. Ed. 2016, 55 (10), 3290-3294.

(32) Wirth, R.; Gao, P.; Nienhaus, G. U.; Sunbul, M.; Jäschke, A. SiRA: A Silicon Rhodamine-Binding Aptamer for Live-Cell SuperResolution RNA Imaging. J. Am. Chem. Soc. 2019, 141 (18), 75627571.

(33) Uno, S. N.; Kamiya, M.; Yoshihara, T.; Sugawara, K.; Okabe, K.; Tarhan, M. C.; Fujita, H.; Funatsu, T.; Okada, Y.; Tobita, S.; Urano, Y. A Spontaneously Blinking Fluorophore Based on Intramolecular Spirocyclization for Live-Cell Super-Resolution Imaging. Nat. Chem. 2014, 6 (8), 681-689.

(34) Grußmayer, K.; Lukes, T.; Lasser, T.; Radenovic, A. SelfBlinking Dyes Unlock High-Order and Multiplane Super-Resolution Optical Fluctuation Imaging. ACS Nano 2020, 14 (7), 9156-9165.

(35) Werther, P.; Yserentant, K.; Braun, F.; Kaltwasser, N.; Popp, C.; Baalmann, M.; Herten, D.-P.; Wombacher, R. Live-Cell Localization Microscopy with a Fluorogenic and Self-Blinking Tetrazine Probe. Angew. Chem., Int. Ed. 2020, 59 (2), 804-810.

(36) Takakura, H.; Zhang, Y.; Erdmann, R. S.; Thompson, A. D.; Lin, Y.; McNellis, B.; Rivera-Molina, F.; Uno, S. N.; Kamiya, M.; Urano, Y.; Rothman, J. E.; Bewersdorf, J.; Schepartz, A.; Toomre, D. Long Time-Lapse Nanoscopy with Spontaneously Blinking Membrane Probes. Nat. Biotechnol. 2017, 35 (8), 773-780.

(37) Dertinger, T.; Colyer, R.; Iyer, G.; Weiss, S.; Enderlein, J. Fast, Background-Free, 3D Super-Resolution Optical Fluctuation Imaging (SOFI). Proc. Natl. Acad. Sci. U. S. A. 2009, 106 (52), 22287-22292.

(38) Lang, K.; Davis, L.; Wallace, S.; Mahesh, M.; Cox, D. J.; Blackman, M. L.; Fox, J. M.; Chin, J. W. Genetic Encoding of Bicyclononynes and Trans-Cyclooctenes for Site-Specific Protein Labeling in Vitro and in Live Mammalian Cells via Rapid Fluorogenic Diels-Alder Reactions. J. Am. Chem. Soc. 2012, 134 (25), 1031710320 .

(39) Darko, A.; Wallace, S.; Dmitrenko, O.; Machovina, M. M.; Mehl, R. A.; Chin, J. W.; Fox, J. M. Conformationally Strained TransCyclooctene with Improved Stability and Excellent Reactivity in Tetrazine Ligation. Chem. Sci. 2014, 5 (10), 3770-3776.

(40) Borrmann, A.; Milles, S.; Plass, T.; Dommerholt, J.; Verkade, J. M. M.; Wießler, M.; Schultz, C.; van Hest, J. C. M.; van Delft, F. L.; Lemke, E. A. Genetic Encoding of a Bicyclo[6.1.0]Nonyne-Charged Amino Acid Enables Fast Cellular Protein Imaging by Metal-Free Ligation. ChemBioChem 2012, 13 (14), 2094-2099.

(41) Werther, P.; Möhler, J. S.; Wombacher, R. A Bifunctional Fluorogenic Rhodamine Probe for Proximity-Induced Bioorthogonal Chemistry. Chem. - Eur. J. 2017, 23 (72), 18216-18224.

(42) Galeta, J.; Dzijak, R.; Obořil, J.; Dračínský, M.; Vrabel, M. A Systematic Study of Coumarin-Tetrazine Light-Up Probes for Bioorthogonal Fluorescence Imaging. Chem. - Eur. J. 2020, 26 (44), 9945-9953.

(43) Pinto-Pacheco, B.; Carbery, W. P.; Khan, S.; Turner, D. B.; Buccella, D. Fluorescence Quenching Effects of Tetrazines and Their Diels-Alder Products: Mechanistic Insight Toward Fluorogenic Efficiency. Angew. Chem., Int. Ed. 2020, 59, 22140-22149.

(44) Schvartz, T.; Aloush, N.; Goliand, I.; Segal, I.; Nachmias, D.; Arbely, E.; Elia, N. Direct Fluorescent-Dye Labeling of $\alpha$-Tubulin in Mammalian Cells for Live Cell and Superresolution Imaging. Mol. Biol. Cell 2017, 28 (21), 2747-2756.

(45) Sauer, M.; Heilemann, M. Single-Molecule Localization Microscopy in Eukaryotes. Chem. Rev. 2017, 117 (11), 7478-7509.
(46) Geissbuehler, S.; Dellagiacoma, C.; Lasser, T. Comparison between SOFI and STORM. Biomed. Opt. Express 2011, 2 (3), 408420 . 